\title{
ANALYSIS OF FRACTIONALLY SPACED WIDELY LINEAR EQUALIZATION OVER FREQUENCY SEleCtive CHANNELS With MUlTiple INTERFERENCES
}

\author{
Hayfa Fhima ${ }^{1,2}$, Daniel Roviras ${ }^{1}$ and Ridha Bouallegue ${ }^{2}$ \\ ${ }^{1}$ CEDRIC/LAETITIA Laboratory, CNAM, Paris, France \\ 2 Innov'COM Laboratory, Sup'Com, Carthage University, Tunisia
}

\begin{abstract}
This paper deals with the performance analysis of both linear (LE) and widely linear (WLE) equalization processes studied in the symbol spaced mode (SSE) as well as in the fractionally spaced one (FSE). This analysis is evaluated - using Matlab software- in terms of bit error rate (BER) and mean square error (MSE) in a system using a rectilinear modulation of type Pulse Amplitude Modulation (PAM) over a frequency selective channel and corrupted by multiple interferences. Moreover, the impact of the number of external interferers on the behavior of the different equalizers is studied. Thus, simulation results show the outstanding performance of the fractionally spaced mode when compared to the symbol spaced one besides the out-performance of the widely linear processing in both modes. Furthermore, results show the performance degradation for the different studied equalizers when the number of interferences increases.
\end{abstract}

\section{KEYWORDS}

Fractionally Spaced Equalizer, widely linear, mean square error, bit error rate, rectilinear modulation, interferences, frequency selective channel.

\section{INTRODUCTION}

It is well known that in wireless communication system, the transmitted signal may not arrive properly at the receiver side. Indeed, du to the wireless propagation channel [1], it may suffer from some deformation and distortion, fading, etc,.. Thus, in order to avoid signal loss at the receiver side, an extra processing should be implemented. This processing is called equalization [2], [3] and it is used in order to suppress interferences and recover the original transmitted signal.

Moreover, the equalization process should take into consideration the characteristics of the signal whether it is second order circular (SOC) or non second order circular (NSOC) [4], [5]. Indeed, it is well known, that for SOC signals, the information is only carried by the auto-correlation function since the pseudo-auto-correlation function is null; hence, the classical linear processing (LE) is sufficient.

However, when the signal is characterized as NSOC, the pseudo-auto-correlation function is nonnull and the information is carried by both the auto-correlation function as well as the pseudo-autocorrelation one [6], [7]. In this latter case, the linear equalization is not an optimal solution and the widely linear (WLE) $[8,9,3,10]$ processing is required and it outperforms the classical one as demonstrated in [1], [4], [11], [12], [13] and [14]. 
International Journal of Wireless \& Mobile Networks (IJWMN) Vol. 11, No. 4, August 2019

On the other hand, it is also known that using fractionally spaced equalizers (FSE) $[15,16,17]$ can enhance the system performance when compared to the symbol spaced equalizers (SSE).

Thus, in this paper, we combine the study of the widely linear equalizer when using the criterion of minimizing the mean square error (MSE) applied in the fractionally spaced mode in a system using a rectilinear modulation corrupted by many external interferences and ISI. Moreover, we study the impact of the number of interferences on the behavior of the FSE. For our knowledge, no such study in literature has been done before.

This paper is organized as follows: A brief overview of related works is presented in Section 2. Then, the system model is introduced in Section 3. Section 4 develops the expressions of MMSELE and MMSE-WLE in both SSE and FSE modes. Simulation results are given in Section 5, followed by the conclusion in Section 6 .

In this article, we use bold capital letters to represent matrix, bold lowercase letters to denote vectors and scalars are represented by lowercase letters. $\mathbb{E}[\cdot]$ is the expectation operator, $(\cdot)^{*},(\cdot)^{T}$ and $(\cdot)^{H}$ denote respectively the conjugate, transpose and the hermitian operations. $\star$ represents the convolution operation.

\section{RELATED WORKS}

The concept of equalization has been widely considered as the main topic in many research articles. Indeed, the necessity of this concept mainly in recovering the transmitted symbols leads to study and analyze the equalization performance according to the signal characteristics. In this context, the out-performance of the widely linear processing and the FSE have encouraged researchers to deal with these two themes. Indeed, authors in [9] have evaluated the performance of both linear (LE) and widely linear (WLE) equalizers in terms of MSE and BER for a system using rectilinear modulation corrupted by inter-symbol-interference (ISI) and external interferences. Besides, authors in [1] have evaluated the MSE in a system where the modulation is improper over a frequency selective channel. In [14], authors, have given an approximation of symbol error rate (SER) with WL receivers in single-input-multiple-output (SIMO) systems in presence of cochannel interferers. In [12], the impact of the number of interferences on the behavior of the equalizers has been highlighted. The performance evaluation studied in [9], [1], [14] and [12], has been done with the use of linear and widely linear equalizers applied on the symbol spaced mode. Regarding the FSE mode, authors in [16], have compared the blind linear equalizer based on two criteria (MMSE as well as zero-forcing). Besides, the convergence rate of the FSE has been studied in [18]. The study of linear fractionally spaced equalizer has been also studied in [19] and [17].

\section{SYSTEM MODEL}

We consider the system model given by Figure 1 with $K$ external interferences. 


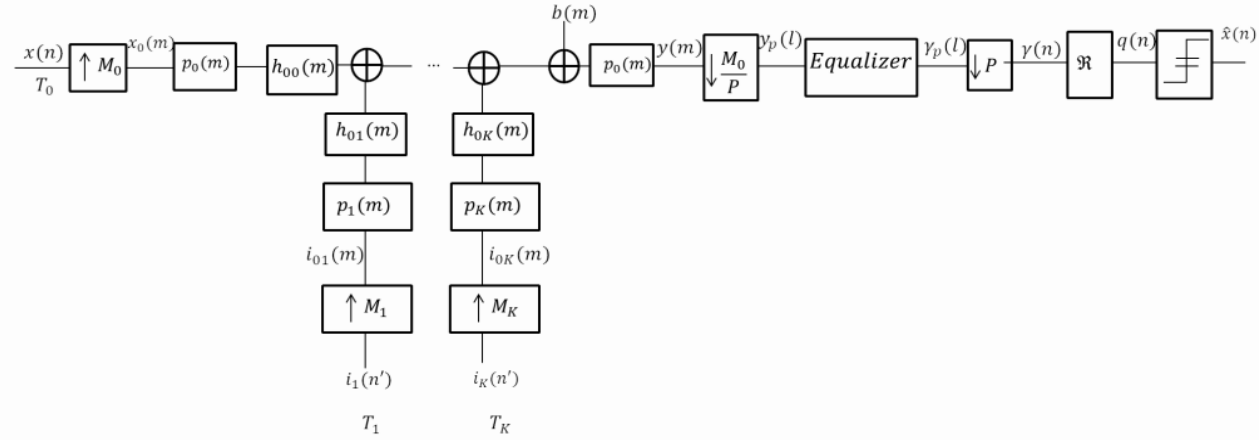

Figure 1: System model with $K$ interferences.

In Figure 1, we transmit a useful rectilinear signal $(x(n))$ (also called signal of interest (SOI)) corrupted by $K$ rectilinear interferences. Without loss of generality, the considered rectilinear modulation is chosen to be an $M-$ ary Pulse Amplitude Modulation ( $M-\mathrm{PAM})$. The PAM signal amplitudes belong to the set $\{ \pm A, \pm 3 A, \ldots, \pm(M-1) A\}$. Thus, the useful $M-$ PAM symbols $x(n)$ are emitted with variance $\sigma_{x}^{2}$ and symbol-duration $T_{0} . x_{0}(m)$ is then a series of Dirac impulses with multiplicative coefficients equal to the $M$-PAM symbols $x(n)$, and spaced by $T_{0}$. This signal is then filtered by the shaping filter with impulse response $p_{0}(m)$ followed by $h_{00}(m)$ which represents the frequency selective channel of the SOI. Likewise, $i_{k}\left(n^{\prime}\right)$, for $k=1, \ldots, K$, represent the $M-\mathrm{PAM}$ symbols transmitted by the $k^{\text {th }}$ interferer, for $k=1, \ldots, K$, with variance $\sigma_{i_{k}}^{2}$ and symbol-duration equal to $T_{k}$. In the following, without loss of generality, we assume that all interferer signals are emitted with the same symbol duration (i.e., $T_{i}=T_{j}, i, j \in[1, K]$ ), and they have the same variance (i.e., $\sigma_{i_{k}}^{2}=\sigma_{i_{k \prime}}^{2}=\sigma_{i}^{2}$ ). Furthermore, We assume that $T_{0}=M_{0} T_{e}$ and $T_{1}=$ $M_{1} T_{e}$, where $T_{e}$ is the sampling time and $M_{1}>M_{0}$. The $k^{t h}$ interferer signal $i_{0 k}(m)$ is then a series of Dirac impulses with multiplicative coefficients equal to the $k^{\text {th }} M-$ PAM interferer symbols, $i_{k}\left(n^{\prime}\right)$, and spaced by $T_{1}$. This signal is also filtered by a shaping filter with impulse response $p_{k}(m)$, followed by a frequency selective channel $h_{0 k}$. The channels output is then corrupted by a circular complex Additive White Gaussian Noise (AWGN) $b(m)$, with variance $\sigma_{b}^{2}$. The receiver front end is composed of a filter matched to the shaping filter of the useful signal, $p_{0}(m)$. The output of this matched filter, $y(m)$, will be sampled with $P$ samples per symbol before processing by the equalizer. Finally, after equalization and decision process, the signal $\hat{x}(n)$ represents the received $M-$ PAM symbols.

The received signal $y(m)$ can be written as follows:

$$
y(m)=p_{0} \star\left[h_{00} \star p_{0} \star x_{0}(m)+\sum_{k=1}^{K} h_{0 k} \star p_{k} \star i_{0 k}(m)+b(m)\right] .
$$

Let us consider $h_{0}(m)=p_{0} \star h_{00} \star p_{0}(m)$ (of length $L_{0}$ ) and $h_{k}(m)=h_{0 k} \star p_{k} \star i_{0 k}(m), k \in$ $1, \ldots, K$ (of length $L_{k}$ ). Eq. (1) becomes equivalent to the following equation:

$$
y(m)=\sum_{l=0}^{L_{0}-1} h_{0}(l) x_{0}(m-l)+\sum_{k=1}^{K} \sum_{l=0}^{L_{k}-1} h_{k}(l) i_{0 k}(m-l)+b(m) .(2)
$$

\section{EXPRESSIONS OF THE EQUALIZERS}

In order to calculate the expressions of the different equalizers, we adopt the criterion of minimizing the mean square error (MSE) between the transmitted signal and the equalized one. We recall that 
International Journal of Wireless \& Mobile Networks (IJWMN) Vol. 11, No. 4, August 2019

there are two types of the MSEs [9], one is used in order to compute the expressions of the equalizers and we note it MSE $\gamma$, and the other one is to evaluate the system performance and we note it MSE $q$. These two MSEs are given as follows:

$$
\begin{aligned}
M S E_{\gamma}= & \mathbb{E}\left[|\gamma(n)-x(n-\Delta)|^{2}\right], \\
& M S E_{q}=\mathbb{E}\left[|\Re(\gamma(n))-x(n-\Delta)|^{2}\right] \\
& =\mathbb{E}\left[|q(n)-x(n-\Delta)|^{2}\right],
\end{aligned}
$$

where $\Delta$ is the delay for the decision procedure [20].

On the other hand, in Figure 1, the down-sampling procedure before equalization process defines whether the equalizers are either implemented in the symbol spaced mode (SSE) or in the fractionally spaced one (FSE). Indeed, by taking $P$ samples per symbol in the equalization process, if $P=1$, then the equalizers are implemented in the SSE mode and if $P>1$ the linear (LE) and the widely linear (WLE) equalizers are called fractionally spaced ones ( $P$ FSE).

It is worth noting that since in the FSE mode we use $P$ times more samples per symbol than in the SSE mode, we have $P$ times more input information in the equalization process. Therefore, the number of the FSE taps is $P$ times higher than the number of the symbol spaced equalizer.

In the following, we note $L_{e}$ the length of the classical linear equalizer (LE). $L_{e}=L_{s}$ when the equalizer is a symbol spaced linear one and $L_{e}=L_{f}$ for the fractionally spaced linear equalizer. Thus, $L_{f}=P L_{s}$. Let us, also, consider the vector $\boldsymbol{y}_{\boldsymbol{p}}(l)$ of length $L_{e}$, defined as $\boldsymbol{y}_{\boldsymbol{p}}(l)=$ $\left[y_{p}\left(l T_{0}\right), y_{p}\left(l T_{0}-T_{e}\right), \ldots, y_{p}\left(l T_{0}-\left(L_{e}-1\right) T_{e}\right)\right]^{T}$.

\subsection{Classical Linear equalizer (LE)}

For the classical linear equalization [1], [9], [21], the equalizer input is the signal $y_{p}(l)$. We recall that the linear process exploits only the information carried by the auto-correlation function of the signal. Thus, its expression is given as follows [9]:

$$
w_{L E}=r_{x y_{p}} R_{y_{p}}^{-1}
$$

where $\boldsymbol{r}_{x \boldsymbol{y}_{\boldsymbol{p}}}=\mathbb{E}\left[x(n) \boldsymbol{y}_{\boldsymbol{p}}^{\boldsymbol{H}}(l)\right]$ is a vector $\in \mathbb{C}^{1 \times L_{e}}$ which represents the inter-correlation vector between the transmitted symbols and the equalizer input. $\boldsymbol{R}_{\boldsymbol{y}_{\boldsymbol{p}}}=\mathbb{E}\left[\boldsymbol{y}_{\boldsymbol{p}}(l) \boldsymbol{y}_{\boldsymbol{p}}^{\boldsymbol{H}}(l)\right]$ represents the autocorrelation matrix $\in \mathbb{C}^{L_{e} \times L_{e}}$ of the equalizer input signal.

Moreover, Eq. (5) can be developed and finally expressed as follows:

$$
\boldsymbol{w}_{\boldsymbol{L E}}=\sigma_{x}^{2} \mathbf{1}_{\Delta} \boldsymbol{H}_{\mathbf{0}}{ }^{H}\left[\sigma_{x}^{2} \boldsymbol{H}_{\mathbf{0}} \boldsymbol{H}_{\mathbf{0}}{ }^{H}+\sum_{k=1}^{K} \sigma_{i}^{2} \boldsymbol{H}_{\boldsymbol{k}} \boldsymbol{H}_{\boldsymbol{k}}{ }^{H}+\sigma_{b}^{2} \boldsymbol{F}_{\mathbf{0}} \boldsymbol{F}_{\mathbf{0}}{ }^{H}\right]^{-1},
$$

where the different matrices and vector, for SSE and FSE respectively, in Eq. 6 are defined as follows:

\section{SSE:}

Let us consider $c_{k}(n)=h_{k}(m)_{\downarrow M_{0}}, k=0, \ldots, K$, which represents the equivalent channel (for SOI when $k=0$ and the $k^{t h}$ interferer when $k=1, \ldots, K$ ) between the transmitter and the receiver in 
International Journal of Wireless \& Mobile Networks (IJWMN) Vol. 11, No. 4, August 2019 the SSE mode. Let us also consider $q_{k}=\operatorname{length}\left(c_{k}(n)\right)-1$.

Therefore, the vector $\mathbf{1}_{\Delta}$ is a vector of length $q_{0}+L_{e}$ defined by:

$$
\begin{gathered}
\mathbf{1}_{\Delta}(l)= \begin{cases}1 & \text { forl }=\Delta \\
0 & \text { else. }\end{cases} \\
\boldsymbol{H}_{\boldsymbol{k}}, \text { for } k=0, \ldots, K, \text { is a matrix } \in \mathbb{C}^{L_{e} \times\left(L_{e}+q_{k}\right)} \text { and is constructed as follows: } \\
\boldsymbol{H}_{\boldsymbol{k}}=\left(\begin{array}{llllllll}
c_{k}(0) & \ldots & c_{k}\left(q_{k}\right) & 0 & 0 & \ldots & 0 \\
0 & c_{k}(0) & \ldots & c_{k}\left(q_{k}\right) & 0 & \ldots & 0 \\
0 & \ldots & 0 & 0 & c_{k}(0) & \ldots & c_{k}\left(q_{k}\right)
\end{array}\right)
\end{gathered}
$$

$\boldsymbol{F}_{\mathbf{0}}$ stands for the correlation matrix of the Noise. It is a function of the received matched filter $p_{0}(m)_{\downarrow M_{0}}$ and it is generated as done for the other matrices $\boldsymbol{H}_{\boldsymbol{k}}$.

\section{P-FSE}

We recall that for the case of the fractionally spaced equalizer, we take $P$ samples per symbol of the received signal in the equalization process. Without loss of generality, we assume that $P=4$. Therefore, as done in the case of SSE, let us consider the following parameters: $c_{k^{\prime}}(l)=h_{k}(m)_{\downarrow} \frac{M_{0}}{P}$, $k=0, \ldots, K . c_{k^{\prime}}(l)$ represents the equivalent channel of the studied system $(k=0$ stands for the SOI, for $k=1, \ldots, K$ it is related to the $k^{t h}$ interferer signal). Hence, $\boldsymbol{H}_{\boldsymbol{k}}, k=0, \ldots, K$ in the FSE mode is a matrix $\in \mathbb{C}^{\left(P \times L_{e}\right) \times\left(L_{e}+q_{k}\right)}$ and is generated as follows:

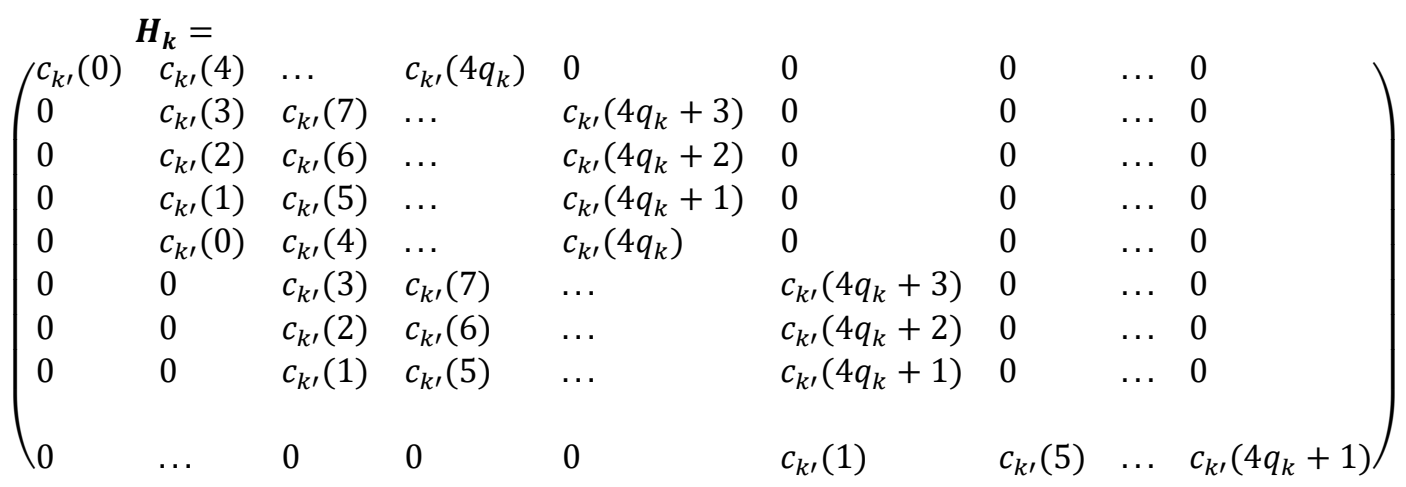

Likewise, the matrix of correlation of the Noise is generated as well as the matrix $\boldsymbol{H}_{\boldsymbol{k}}$.

\subsection{Widely linear equalizer (WLE)}

Unlike the classical linear processing, the widely linear equalization (WLE) [1], [8], [3], [11] exploits the information carried by both the auto-correlation function and the pseudo-autocorrelation one, meaning that it uses the information contained in the second order properties of the signal.

Since the widely linear processing is applied on the received signal as well as its complex conjugate version, let us consider the following vector $\widetilde{\boldsymbol{y}}_{\boldsymbol{p}}(l)$ of length $2 L_{e}$ and defined as: $\widetilde{\boldsymbol{y}}_{\boldsymbol{p}}(l)=$ $\left[\boldsymbol{y}_{\boldsymbol{p}}^{\boldsymbol{T}}(l), \boldsymbol{y}_{\boldsymbol{p}}^{*}(l)\right]^{T}$ which represents the WL equalizer input. 
International Journal of Wireless \& Mobile Networks (IJWMN) Vol. 11, No. 4, August 2019

The expression of the widely linear equalizer is given by the following expression [11]:

$$
\widetilde{w}_{W L}=r_{x \widetilde{y}_{p}} R_{\widetilde{y}_{p}}^{-1}
$$

where $\boldsymbol{r}_{x \tilde{\boldsymbol{y}}_{p}}$ is a vector $\in \mathbb{C}^{1 \times 2 L_{e}}$ and represents the inter-correlation vector between the transmitted symbols and the equalizer input in its widely linear version (i,e: $\widetilde{\boldsymbol{y}}_{\boldsymbol{p}}(l)$ ). Besides, $\boldsymbol{R}_{\widetilde{\boldsymbol{y}}_{\boldsymbol{p}}}$ is a matrix $\in \mathbb{C}^{2 L_{e} \times 2 L_{e}}$, called the auto-correlation matrix of the signal $\widetilde{\boldsymbol{y}}_{\boldsymbol{p}}(l)$.

As done in the classical linear case, Eq. (9) can be developed and expressed as follows:

$$
\boldsymbol{W}_{\boldsymbol{W L E}}=\sigma_{\chi}^{2} \mathbf{1}_{\Delta} \widetilde{\boldsymbol{H}}_{\mathbf{0}}{ }^{H}\left[\sigma_{\chi}^{2} \widetilde{\boldsymbol{H}}_{\mathbf{0}} \widetilde{\boldsymbol{H}}_{\mathbf{0}}{ }^{H}+\sum_{k=1}^{K} \sigma_{i}^{2} \widetilde{\boldsymbol{H}}_{\boldsymbol{k}} \widetilde{\boldsymbol{H}}_{\boldsymbol{k}}{ }^{H}+\sigma_{b}^{2} \widetilde{\boldsymbol{F}}_{\mathbf{0}} \widetilde{\boldsymbol{F}}_{\mathbf{0}}{ }^{H}\right]^{-1},
$$

where $\widetilde{\boldsymbol{H}}_{\boldsymbol{k}}=\left[\begin{array}{l}\boldsymbol{H}_{\boldsymbol{k}} \\ \boldsymbol{H}_{\boldsymbol{k}}{ }^{*}\end{array}\right], k=0, \ldots, K$ and $\widetilde{\boldsymbol{F}}_{\mathbf{0}}=\left[\begin{array}{ll}\boldsymbol{F}_{\mathbf{0}} & \mathbf{0} \\ \mathbf{0} & \boldsymbol{F}_{\mathbf{0}}{ }^{*}\end{array}\right]$, and $\boldsymbol{H}_{\boldsymbol{k}}$ and $\boldsymbol{F}_{\mathbf{0}}$ are the matrix defined in the previous item.

It is worth to mention that Eq. (10) stands valid for SSE mode as well as the FSE one.

\section{Simulation RESULTS}

In this section, the performance evaluation of the linear (LE) (Eq. (6)) and widely linear (WLE) (Eq. (10)) equalizers for both SSE and FSE mode is studied. Besides, the impact of the number of the external interferences on the equalizers' behavior is analyzed. The different simulation realizations are done using Matlab software.

Thus, for these simulations, the different shaping filters (see Figure 1) are chosen to be square root raised cosine (SRRC) filters with a roll off factor equal to 1. Regarding the symbol durations, all the interferences have the same symbol duration. Without loss of generality, the interferer symbol duration is chosen to be twice the SOI one (i.e., $T_{1}=2 T_{0}$ ). The different channel impulse responses of all signals (SOI + interferers) have 60 taps, having each one a Rayleigh modulus and a phase uniformly distributed in $[0,2 \pi]$. Likewise, these taps are kept constant during the transmission of $10^{4} M$-PAM symbols for $M=2$ as well as for $M=16$. The length of the classical linear equalizer in the SSE mode is equal to $L_{e}=15$ taps; thus, the linear equalizer in the FSE mode has $4 \times L_{e}=$ 60 taps. The presented simulation results are the average of 100 different channel realizations.

Figures 2 and 3 compare the system performance of both linear and widely linear equalizers implemented in the SSE mode as well as in the FSE one. This comparison is evaluated in terms of MSE and BER in a system where only one interferer is present $(K=1)$ and using respectively 2 -PAM modulation for Figure 2 and 16 -PAM modulation for Figure 3. 


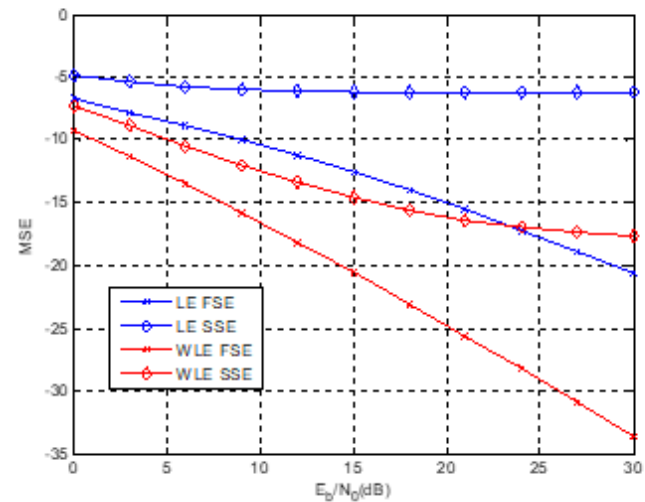

(a) MSE

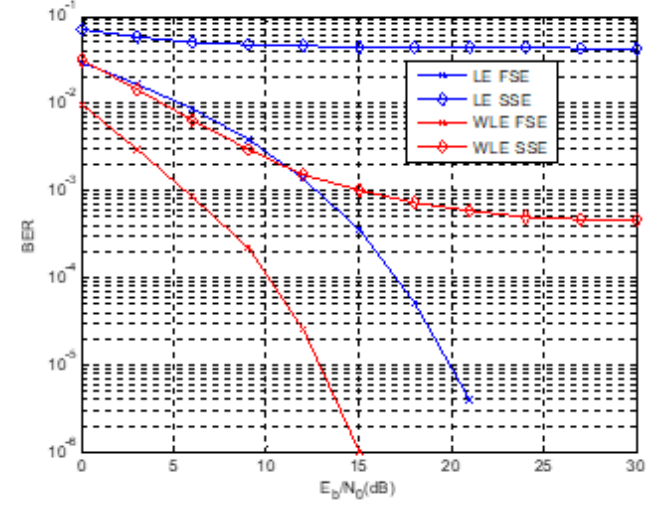

(b) BER

Figure 2: Error performance for $K=1$ using $2-P A M$ modulation.

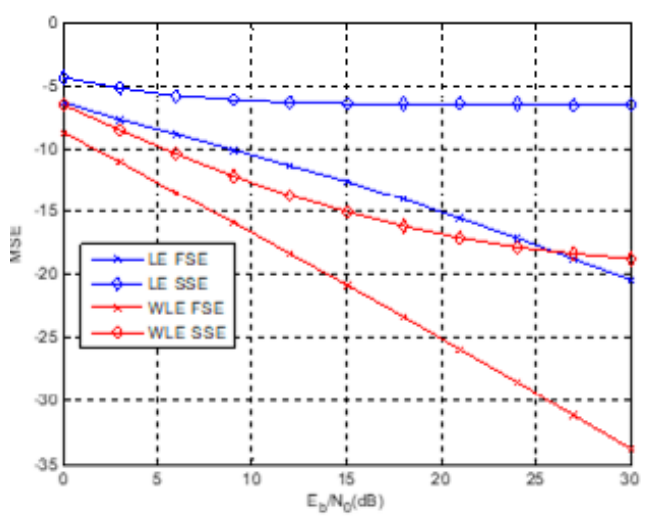

(a) MSE

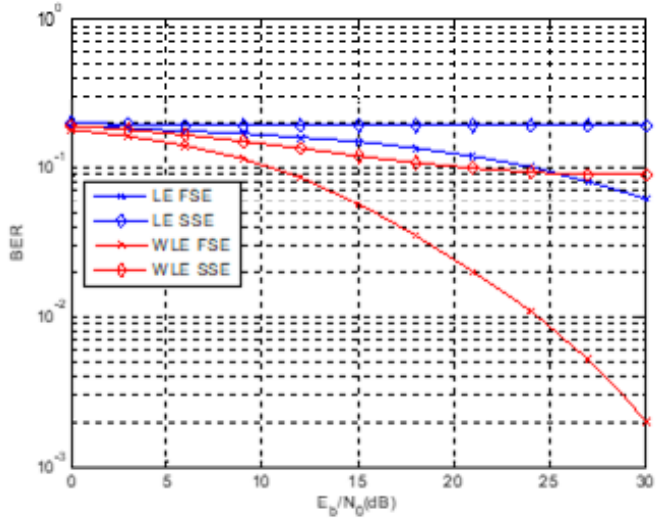

(b) BER

Figure 3: Error performance for $K=1$ using 16 -PAM modulation.

From results depicted in Figures 2 and 3, we can deduce that the widely linear equalizer (WLE) outperforms the classical linear one (LE) and that for both modes: SSE and FSE. Indeed, this outperformance can be explained via two reasons. The first one is the non-circularity characteristic of the transmitted signal. The second one is related to the presence of the inter-symbol-interferences (ISI) caused by the frequency selective channels.

Moreover, the equalizers implemented in the FSE mode give better performance when compared to the SSE mode. Indeed, the fractionally equalizers input signals use more information than the SSE leading to a better performance. Furthermore, the worst behavior is given by the classical linear equalizer used in the SSE mode, where it can not recover the transmitted symbols.

Focusing on the analysis of the fractionally spaced equalizers behavior as a function of the number of external interferers $K$, Figures 4 and 5 present the impact of $K$ on the equalizers performance in terms of MSE and BER in presence of 1 interferer $(K=1)$ and more than one interferer $(K=3)$ and that using respectively, $2-$ PAM (Figure 4) and 16 -PAM modulation (Figure 5). 
International Journal of Wireless \& Mobile Networks (IJWMN) Vol. 11, No. 4, August 2019

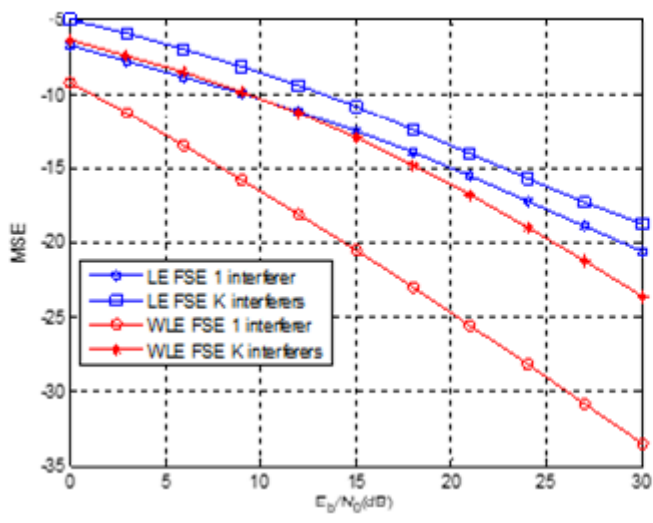

(a) MSE

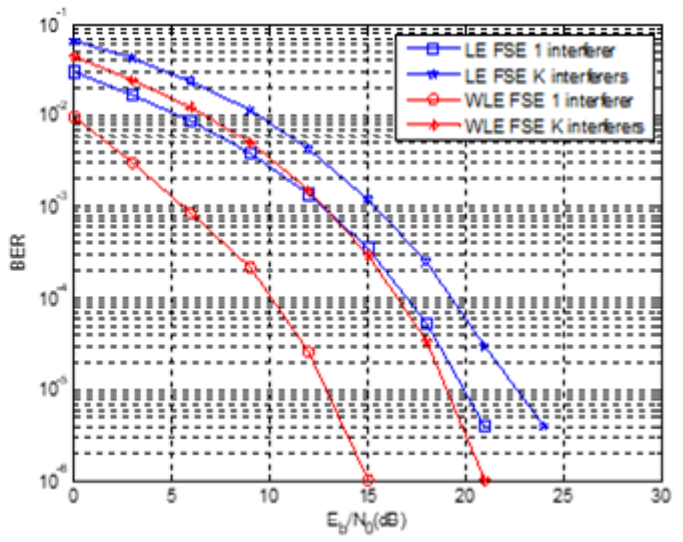

(b) BER

Figure 4: Impact of $K$ on fractionally spaced equalizers performance for 2 -PAM modulation.

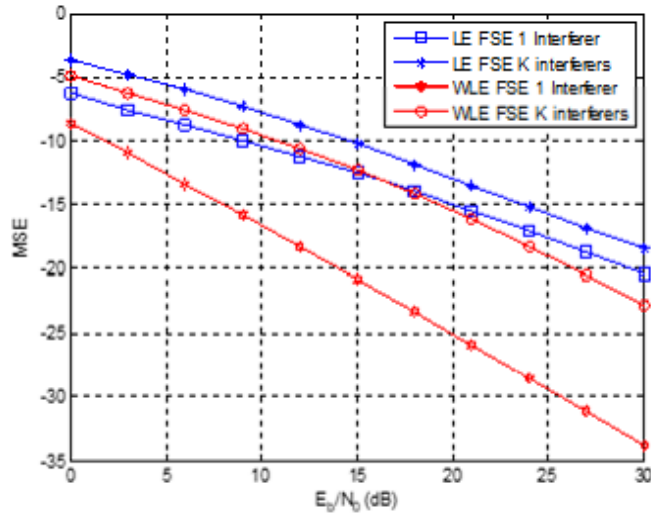

(a) MSE

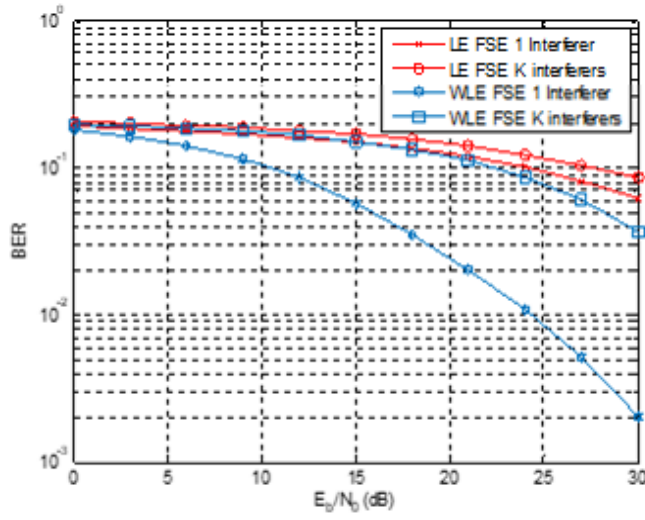

(b) BER

Figure 5: Impact of $K$ on fractionally spaced equalizers performance for 16 -PAM modulation.

Results depicted from Figures 4 and 5, show the out-performance of the widely linear equalizer when compared to the classical one in presence of $K=1$ and $K=3$ interferences and for 2 -PAM and $16-\mathrm{PAM}$ modulations. Furthermore, $\forall M$, when the number of interferences $(K)$ increases, the performances of the fractionally equalizers (LE and WLE) decrease. Besides, the worst performance is provided by the linear equalizer for any PAM order modulation $(\forall M)$ and $\forall K$.

\section{CONCLUSION}

In this article, we have analyzed the performance of both linear and widely linear equalizers implemented in the symbol spaced mode and the fractionally spaced one. These equalizers are studied in a system using rectilinear modulation of type $M$-PAM modulation, over frequency selective channels and in presence of $K$ NSOC interferers. We have shown that the fractionally spaced widely linear processing provides the best performance $-\forall K$ and $\forall M$ - when compared to the classical linear equalization process. Moreover, we have shown that when the number of interferences $(K)$ increases, the performances of the fractionally spaced equalizers decrease but they still provide better performance than the symbol spaced equalizers. 
International Journal of Wireless \& Mobile Networks (IJWMN) Vol. 11, No. 4, August 2019

\section{ACKNOWLEDGMENT}

This work was supported by the WONG5 Project from the French National Agency (ANR) under Contract ANR-15-CE25- 0005-02.

\section{REFERENCES}

[1] W.H. Gerstacker, R. Schober and A. Lampe, “'Receivers With Widely Linear Processing for FrequencySelective Channels," IEEE Transactions on Communications, vol. 51, no. 9, pp. 1512-1523, 2003.

[2] R. K.Mohammed, “Comparing various channel estimation techniques for OFDM systems using Matlab", International Journal of Wireless \& Mobile Networks (IJWMN) Vol. 11, No. 3, June 2019.

[3] B. Picinbono and P. Chevalier, "Widely Linear Estimation with Complex Data," IEEE Transactions on Signal Processing, vol. 43, no. 8, pp. 2030-2033, 1995.

[4] P. Chevalier and F. Pipon, “New Insights Into Optimal Widely Linear Array Receivers for the Demodulation of BPSK, MSK, and GMSK Signals Corrupted by Noncircular Interferences Application to SAIC," IEEE Transactions on Signal Processing, vol. 54, no. 3, pp. 870-883, Mar. 2006.

[5] J.P. Delmas, A. Oukaci, and P. Chevalier, "Performance analysis of the GLRT-based array receivers for the detection of a known signal corrupted by noncircular interference", in International Conference on Acoustics, Speech and Signal Processing (ICASSP 2010), Dallas, March 2010.

[6] P. Schreier, L. Scharf, and C. T. Mullis, “Detection and Estimation of Improper Complex Random Signals," IEEE Transactions on Information Theory, vol. 51, no. 1, pp. 306-312, Aug. 2005.

[7] B. Picinbono, “'On circularity," IEEE Transactions on Signal Processing, vol. 45, no. 9, pp. 3473-3482, Dec. 1994.

[8] E.P.da Silva, D. Zibar, “'Widely Linear Equalization for IQ Imbalance and Skew Compensation in Optical Coherent Receivers, Journal of Lightwave Technology, vol. 34, Aug. 2016.

[9] H. Fhima, H. Shaiek, R. Zayani, D. Roviras, B. S. Chang, and R. Bouallegue. " Analysis of widely linear equalization over frequency selective channels with multiple interferences". In 2018 14th International Conference on Wireless and Mobile Computing, Networking and Communications (WiMob), pages 83-88, Oct 2018.

[10] X. Wu, Y. Cai, M. Zhao, R.C. DE Lamare and B. Champagne, “'Adaptive Widely Linear Constrained Constant Modulus Reduced-Rank Beamforming", in IEEE Transactions on Aerospace and Electronic Systems VOL. 53, NO. 1 Feb. 2017.

[11] B. Chang, C. da Rocha, D. Ruyet, and D. Roviras, “Widely linear MMSE precoding and equalization techniques for SC-FDE systems," EURASIP Journal on Advances in Signal Processing, vol. 2014, no. 1, p. 124, 2014.

[12] H. Fhima, R. Zayani, H. Shaiek, D. Roviras, B.S. Chang, and R. Bouallegue, 'Widely linear equalizer performance with multiple independent interferences", in accepted to ISCC 2017. IEEE, 2017.

[13] N. Song, R.C. de Lamare, M. Haardt and M. Wolf,“'Adaptive Widely Linear Reduced-Rank Interference Suppression Based on the Multistage Wiener Filter", in IEEE Transactions Signal Processing, vol. 60, no. 8, pp. 4003-4016, Aug. 2012.

[14] K. Kuchi and V.K. Prabhu , “Performance Evaluation for Widely Linear Demodulation of PAM/QAM Signals in the Presence of Rayleigh Fading and Co-channel Interference", in IEEE Transactions on 
International Journal of Wireless \& Mobile Networks (IJWMN) Vol. 11, No. 4, August 2019 communications, vol. 57, no. 1, January 2009.

[15] J.R. TREICHLER, I. FIJALKOW, and C.R. JOHNSON, JR, “'Fractionally Spaced Equalizers.", in IEEE SIGNAL PROCESSING MAGAZINE, May 1996.

[16] Z. Zhang, L. Zhang, P. Shui, "Fractionally spaced equalizer based on multichannel linear prediction", in Proceedings of the IEEE 6th Circuits and Systems Symposium on Emerging Technologies: Frontiers of Mobile and Wireless Communication (IEEE Cat. No.04EX710), 2004.

[17] M. R. Heidarpour, M. Uysal and M. O. Damen, 'Design and Analysis of Broadband Amplify-andForward Cooperative Systems: A Fractionally-Spaced Sampling Approach", IEEE Transactions on Signal Processing, vol. 64, pp. 4936-4951, Oct.2016.

[18] S. S. Ranhotra and A. Mishra, “'On the Performance of Fractionally Spaced and Constant Modulus Equalizer", in 2015 IEEE International Conference on Computational Intelligence \& Communication Technology,2015.

[19] L. Zhiyuan, L. Baojin and Lou Peide, “'Linear Time And Frequency Domain Fractionally Spaced Equalization", in 2007 International Symposium on Microwave, Antenna, Propagation and EMC Technologies for Wireless Communications, 2007.

[20] P.A. Voois, I. Lee, and J.M. Cioffi, “The effect of decision delay in finite length decision feedback equalization,IEEE Transactions on Information Theory", vol. 42, pp. 618-621, Mar. 1996.

[21] A. Ikhlef and J. Louveaux, “An Enhanced MMSE per subchannel Equalizer for highly Frequency Selective Channels for FBMC/OQAM systems", in Signal Processing Advances in Wireless Communications, 2009. SPAWC'09., 2009.

\section{AUTHORS}

HAYFA FHIMA received the Engineer degree from High School of Communications of Tunis (SUP'COM) in 2012. She is currently a PhD student at SUP'COM and the Conservatoire National des Arts et des Metiers (CNAM) in Paris. Her research interests include signal processing for digital communications, equalization, widely linear processing and FBMC-OQAM systems.

DANIEL ROVIRAS was born in 1958. He received the Engineer degree from SUPELEC, Paris, France, in 1981, and the Ph.D. degree from the National Polytechnic Institute of Toulouse, Toulouse, France, in 1989. He spent in the industry as a Research Engineer for seven years. He joined the Electronics Laboratory, Ecole Nationale Supérieure d'Electrotechnique, d'Electronique, d'Informatique, et des Télécommunications (ENSEEIHT). In 1992, he joined the Engineering School, ENSEEIHT, as an Assistant Professor, where he has been a Full Professor since 1999. Since 2008, he has been a Professor with the Conservatoire National des Arts et Métiers (CNAM), Paris, France, where his teaching activities are related to radio-communication systems. He is currently a member of the CEDRIC Laboratory, CNAM. His research activity was first centered around transmission systems based on infrared links. Since 1992, his topics have widened to more general communication systems, such as mobile and satellite communications systems, equalization, and predistortion of nonlinear amplifiers, and multicarrier systems. 
International Journal of Wireless \& Mobile Networks (IJWMN) Vol. 11, No. 4, August 2019

RIDHA BOUALLEGUE received the PhD degree and the HDR degreein multiuser detectionin cellular radio systems of the next generation from the University of Tunis El Manar, Tunis, Tunisia, in 1998 and 2003, respectively. In 2005, he was a Director with the Research Unit Telecommunications Systems and the National Engineering School of Sousse, Sousse, Tunisia. He is currently a Professor with the National Engineering School of Tunis, Tunis, Tunisia, and with the Higher School of Communications of Tunis, University of Carthage, Tunis. His research interests include the physical layer of telecommunication systems, in particular on digital communications systems, multiple input multiple output systems, output orthogonal frequency-division multiplexing, code-division multiple access, ultra-wideband, WiMAX, Long-Term Evolution, software-defined radio, etc. He is the author of two book chapters, 75 articles in refereed conference lectures, and 15 journal articles. 